\title{
Analysis of energy characteristics of acoustic emission signals during uniaxial compression of geomaterial samples
}

\author{
Vladimir Sychev ${ }^{1 *}$, Leonid Bogomolov ${ }^{2}$, Dmitriy Kulkov ${ }^{1}$ \\ ${ }^{1}$ Science Research Station of the RAS in Bishkek city, Kyrgyzstan \\ ${ }^{2}$ Institute of Marine Geology and Geophysics FEBRAS, Russia
}

\begin{abstract}
Acoustic emission (AE) signals were obtained during deformation by uniaxial compression of specimens of various geomaterials. Experiments on uniaxial compression were carried out on a low-noise lever setup with water leakage, where the maximum load on the sample does not exceed $250 \mathrm{kN}$. The received signals were digitized by an 8-channel USB 3000 ADC unit with a width of 14 bits and a maximum sampling rate of $3 \mathrm{MHz}$. The energy distribution functions of AE signals are considered. The maximum amplitude of the $\mathrm{AE}$ waveform was selected as the energy characteristic of the $\mathrm{AE}$ signal. The flow of $\mathrm{AE}$ events is considered from the viewpoint of nonequilibrium thermodynamics using the Tsallis statistics. To describe the energy distribution function of the $\mathrm{AE}$ signals, we used a modified model of a stick-slip earthquake source "discontinuous sliding" of two plates over each other along a fault in the presence of friction and the principle of maximum entropy. The model is used to quantify long-range correlations arising in the flow of earthquakes. It is shown that the AE signal flow is a system with memory and longrange correlations. The analysis of the behavior of the Tsallis parameter was carried out throughout the experiment.
\end{abstract}

\section{Introduction}

The similarity of the processes occurring in geological structures in the Crust, and deformation that in the rocks tested in laboratory experiments $[1-6]$, creates a prerequisite to study the deep geostraing processes using the method of physical modeling on rocks specimens. Modeling of inelastic deformation and fracture of specimens in experiments on rheological presses has already demonstrated its effectiveness for a number of seismological and tectonophysical problems [7 - 10]. The data on acoustic emission are informative [11 - 12] as a monitoring tool of internal changes in opaque materials specimen at different stages of loading (up to their macrofracture).

The application of acoustic emission AE in experiments on physical modeling of the seismic process (or its elements) is based on the concept of self-similarity of the geological environment, including the similarity of damage accumulation characteristics on different

* Corresponding author: koitash@mail.ru 
spatio-temporal scales [13 - 16]. The source of most acoustic impulses (AE signals) is the appearance or increase in the length of a microcrack in the rock samples with a brittle or semi-brittle destruction type. The revealed patterns in the signal sequence, reflecting and accompanying changes in the state of the material during fracturing, are transferred to the full-scale geophysical (seismological) scale. The adequacy of this schematically presented approach was confirmed already by the results of the first works [17 - 19], devoted to the destruction of homogeneous or inhomogeneous materials under uniaxial compression. Subsequent studies of acoustic emission, carried out with the use of more advanced technical means for recording AE, again demonstrated the possibility of obtaining results adequate to the conditions in seismogenic zones [20 - 23]. Taking this into account, it is quite natural to use continuous $\mathrm{AE}$ measurements when studying the process of rock cracking, which is caused not only by uniaxial mechanical compression, but also by the additional effect of model sources of vibrations and electromagnetic fields.

The fundamental laws of the flow of seismic events have been established in the seismology. The most famous relationship is the Gutenberg - Richter energy distribution law for earthquakes [24]:

$$
P(E) \sim E^{-\gamma},
$$

where $\gamma \approx 5 / 3, E$ is the earthquake energy [25]. In terms of the number of events with a magnitude $M$ exceeding $m$, the distribution law takes the form:

$$
N(M>m) \sim 10^{-\mathrm{bm}},
$$

where $N$ - denotes the number of events for a specified fixed time period and in a given geographic region, $b$ - a constant ( $b$-value), in most cases takes a value of about 0.9 [26].

It should be noted that the laws describing earthquake sequences are mainly empirical and cannot be derived from the provisions of equilibrium (classical) thermodynamics. Classical thermodynamics is based on the concept of entropy, which is a function of the state of a thermodynamic system. If $p_{i}$ is the probability of stay systems in state with number $i(i=1$, $\left.\ldots, N, \sum_{i=1}^{N} p_{i}=1\right)$, then the Boltzmann - Gibbs entropy is determined by the standard formula for calculating the statistical mechanical entropy of a thermodynamic system [27]:

$$
S=-k \sum_{i=1}^{N} p_{i} \ln p_{i}
$$

where $\mathrm{k}$ is the Boltzmann constant $\left(k=1.38 \cdot 10^{-23} \mathrm{~J} / \mathrm{K}\right), N$ is the number of possible states of the system. The total entropy of the system is equal to the sum of the entropy of its parts in this case (property of additivity of entropy). In particular, if the system consists of two independent subsystems $A$ and $B$, then the total entropy of the system will be as follows:

$$
S(A+B)=S(A)+S(B)
$$

In 1988, Constantino Tsallis, to describe complex non-additive statistical systems, generalized the classical Boltzmann-Gibbs formula by introducing into the expression the parameter q, which characterizes the degree of non-additivity. He proposed the so-called nonextensive or non-additive entropy [28], which on a discrete number of microstates $\mathrm{N}$ is determined as follows expression:

$$
S_{q}=k \frac{1}{q-1}\left(1-\sum_{i=1}^{N} p_{i}^{q}\right) ; \quad \sum_{i=1}^{N} p_{i}=1
$$


where $p_{i}$ is the probability that the system is in the $i$ - state, $N$ is the number of states of the system, $k$ is some positive constant that determines the unit of measurement of entropy and in physical formulas serves for a bundle of dimensions, such as the Boltzmann constant.Boltzmann statistics, expression (3), corresponds to the limit $q \rightarrow 1$. And $q>1$ indicates the presence of long-range correlations and memory in a nonequilibrium system when additivity is violated.Thus, the Tsallis entropy is no longer an extensive function. If, for example, the system is divided into two independent subsystems $A$ and $B$, then the total entropy of the system will be as follows:

$$
S_{q}(A+B)=S_{q}(A)+S_{q}(B)+(1-q) S_{q}(A) S_{q}(B)
$$

The parameter $\mathrm{q}$, in this case, is a measure of the nonextensiveness of the system under consideration: for $q<1$, we obtain the over-extensive case, $q=1$ - the extensive case, and for $q>1$ - the sub-extensive case. [29].

These provisions of nonequilibrium thermodynamics are increasingly used to describe the flow of earthquakes - a non-extensive analysis of the flow of seismic events [30 -32].

The analysis of the flow of earthquakes, as an open nonequilibrium system, is based on the function of the distribution of earthquakes by energy (Fragment-Asperity Interaction Model for Earthquakes) proposed in [33] and based on the well-known model of stick-slip earthquakes - "intermittent sliding" of two plates over each other along fracture in the presence of friction [34] and the principles of non-intensive statistical physics.

Using this model, and the principle of maximum entropy in [33], an expression was obtained for the function of the distribution of earthquakes by energy, which generalizes the original function of the distribution of earthquakes by the Gutenberg - Richter energies. In turn, in [35] this model was refined using a more realistic relationship between the earthquake energy and the fragment size. Further, already in [36], an analytical expression was obtained that describes the generalized Gutenberg - Richter law, which relates the cumulative number of earthquakes with a magnitude exceeding the threshold value with the Tsallis parameter $q$ :

$$
\log \left(\frac{N\left(M>M_{t h}\right)}{N}\right)=\left(\frac{2-q}{1-q}\right) \log \left[1-\left(\frac{1-q}{2-q}\right)\left(\frac{10^{M_{t h}}}{a^{2 / 3}}\right)\right]
$$

where $N\left(M>M_{t h}\right)$ is the number of earthquakes with energies greater than the threshold value $M_{t h}$, and $M \sim \log (E), E$ is the earthquake energy, $N$ is the total number of earthquakes, a is the proportionality constant between the earthquake energy $E$ and the size of the fragment of blocks $r^{3}$ between faults and has the dimension of volumetric energy density [35 - 38].

The above expression describes well the energy distribution of earthquakes in the entire range of magnitudes, in contrast to the Gutenberg - Richter empirical formula [35].Moreover, the estimate of the Tsallis parameter $q$ turns out to be stable with respect to the choice of the threshold value of the magnitude $M_{t h}$, rather than the estimate of the bvalue parameter, which is more demanding on the choice of the linear portion of the repeatability graph [38]. Equation (7) allows us to estimate the degree of nonextensiveness in the region under consideration.

Many publications indicate that the value of the $q$ parameter can be used as a measure of the stability of the active tectonic zone [38 - 40]. A sharp increase in the parameter $q$ indicates an increase in the interaction between fault blocks and their fragments and implies a deviation from the equilibrium state [38].

When carrying out experiments on the destruction of various materials on the behavior of acoustic emission parameters, researchers are trying to establish some regularities in the description of the AE event distribution function by energy. First of all, the energy distribution of the registered $\mathrm{AE}$ events is the determination of the b-value parameter. An attempt is made to describe the distribution function of the AE signal energies by various 
dependences: at the initial stage of loading, it is exponential, and at a stage close to failure, it is a power law [41]. The application of non-extensive analysis in this case is extremely rare $[42,43]$. The purpose of this work is precisely to attempt to describe the distribution function of $\mathrm{AE}$ events from the point of view of nonequilibrium thermodynamics using the Tsallis statistics.

\section{Equipment and technique}

Waveforms of acoustic emission (AE) signals are recorded during deformation of samples of various geomaterials under uniaxial compression. Experiments on uniaxial compression are carried out on a low-noise lever setup with water leakage [44], where the maximum load on the sample does not exceed $250 \mathrm{kN}$. All tested samples were made in the form of a rectangular parallelepiped, sandstone with a square section, dimensions $25 \mathrm{~mm} \mathrm{x}$ $25 \mathrm{~mm} \times 60 \mathrm{~mm}(\mathrm{~L} \times \mathrm{W} \times \mathrm{H})$, and granite and marble with a rectangular section, dimensions $\mathrm{L} \times \mathrm{W} \times \mathrm{H}(40 \mathrm{~cm} \times 20 \mathrm{~cm} \times 80 \mathrm{~cm}$.). In fig. 1 shows examples of tested samples of sandstone (a), marble and granite (b) and a lever arrangement (c).

a

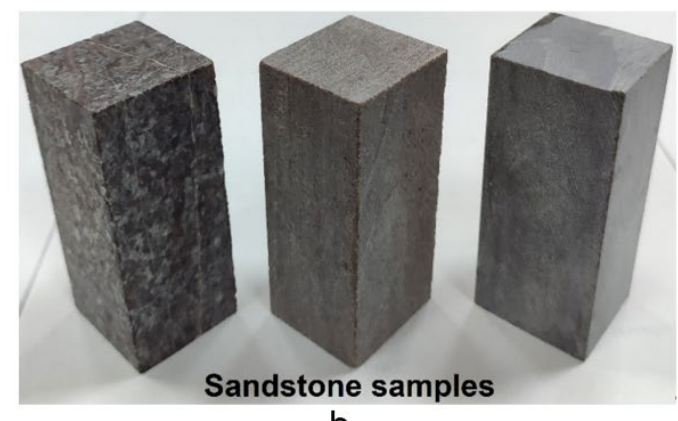

b

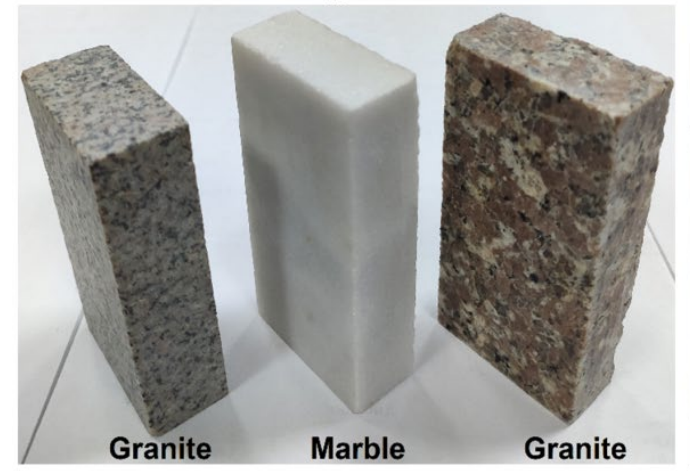

C

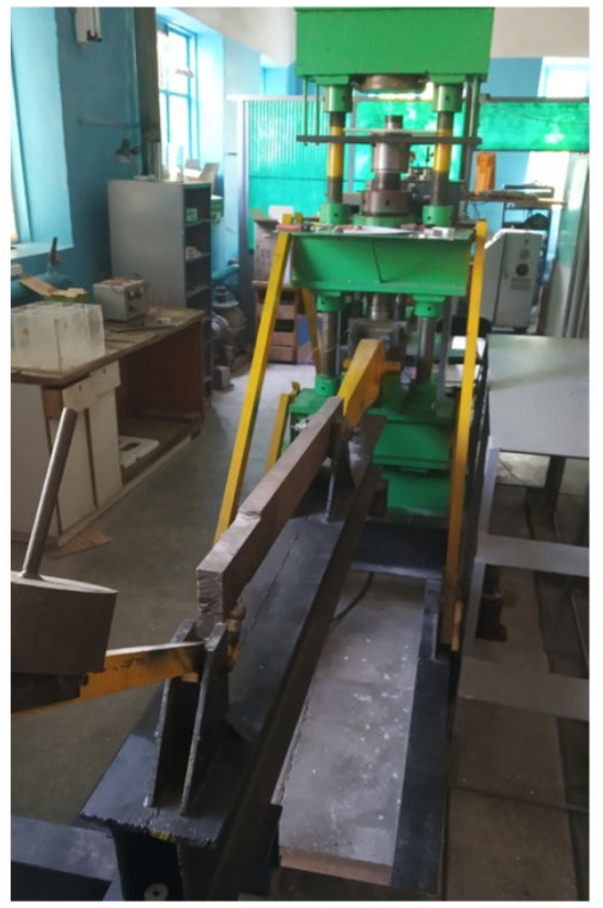

Fig. 1. Examples of samples of geomaterial: sandstone, a) granite and marble b). Lever system front view c)

The experiments were carried out with a linearly increasing load and a stepwise change in load. Examples of load change shares shown in Fig. 2. 
a

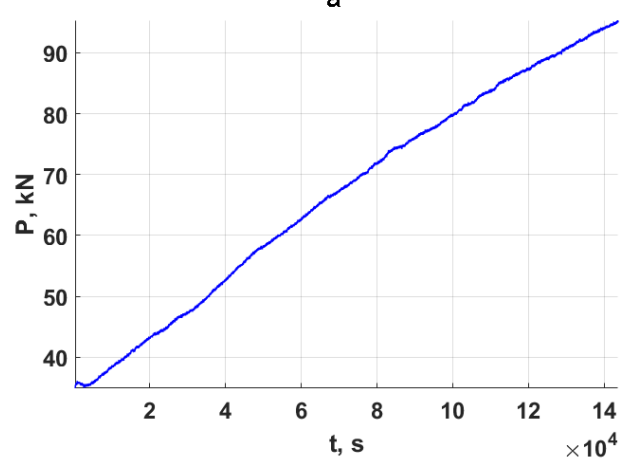

b

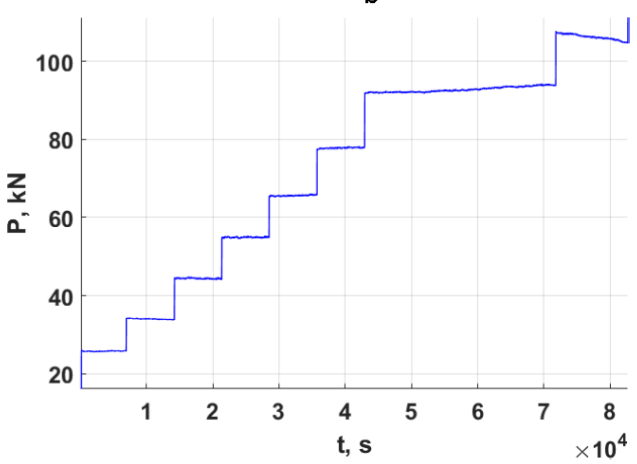

Fig. 2. Examples of linear a) and step b) load changes.

To register AE signals, SE-2MEG-P and (DECI, USA) sensors were used, with an operating frequency range from $200 \mathrm{kHz}$ to $2 \mathrm{MHz}$, fixed on the test sample of geomaterial. The received signals were digitized by an 8-channel USB 3000 ADC unit with a width of 14 bits and a maximum sampling rate of $3 \mathrm{MHz}$.Registration of AE signals is carried out in standby mode, provided that the signal amplitude exceeds the set discriminator threshold and during the time interval determined by the ADC memory buffer volume and the signal sampling period. In the course of the experiment, the waveforms of the AE signals were recorded and saved on the hard disk of the recording computer for subsequent processing.

\section{Results and discussion}

During the experiment, a large number (from 15,000 to 400,000) of various AE signals are recorded, among which there are useful signals, noise, and their combinations [45]. For rejection of AE signals, the algorithm [45] was implemented, consisting of two stages: 1selection of a useful signal in the amplitude-frequency spectrum in the operating range of the sensor (from 0 to $200 \mathrm{kHz}$ ) according to its calibration curve; 2- analysis of the amplitude-frequency spectrum of signals, in which the ratios of the sums of the amplitudefrequency spectrum were calculated in the range from the lower limit of sensor sensitivity $(200 \mathrm{kHz})$ to the Nyquist frequency, based on the ADC sampling limit $(1.5 \mathrm{MHz})$ to the sum of amplitudes in the spectrum of the entire signal.In the array of AE signal waveforms formed in this way, the maximum amplitude of the AE waveform was selected as the energy characteristic, and a catalog of AE events was formed. An example of the energy distribution of AE signals for two experimental sessions is shown in Fig. 3 

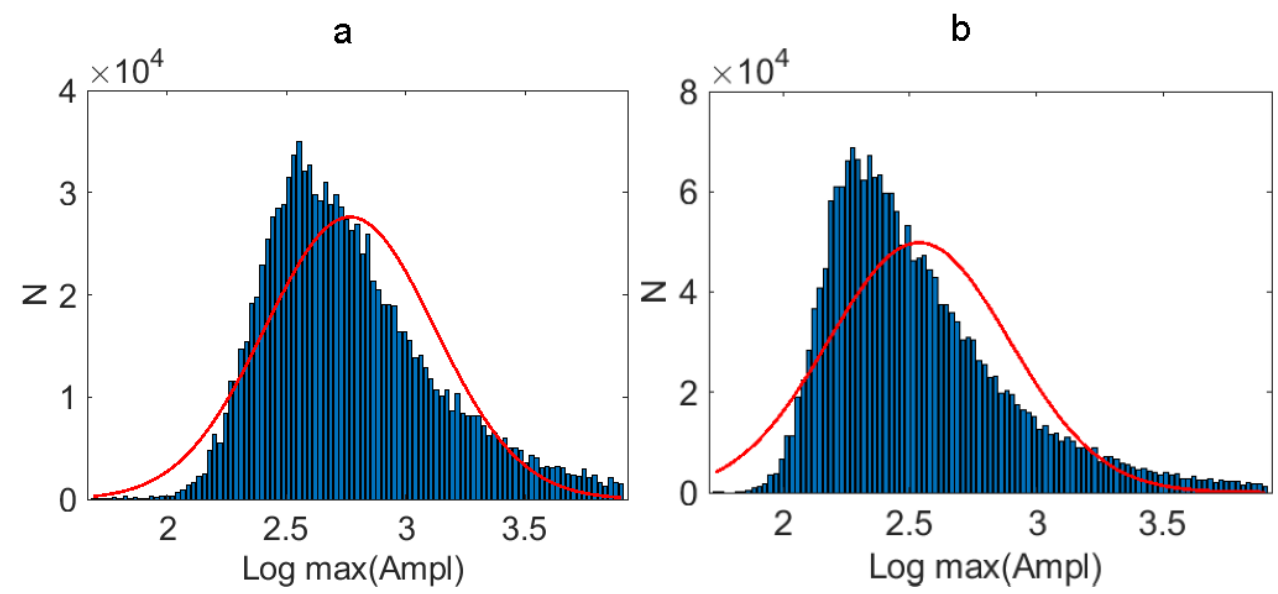

Fig. 3. Examples of energy distribution functions of AE signals with linear a) and step b) load change and approximation by a normal distribution function.

It is seen that such distributions have heavy tails and are not described by a Gaussian function. Let us describe these energy distribution functions of events using expression (7) Fig. 4. The figure shows the energy distribution of $\mathrm{AE}$ events, their approximation using expression (7), the obtained values of the Tsallis parameters $q$ and a, as well as the errors in determining these quantities.

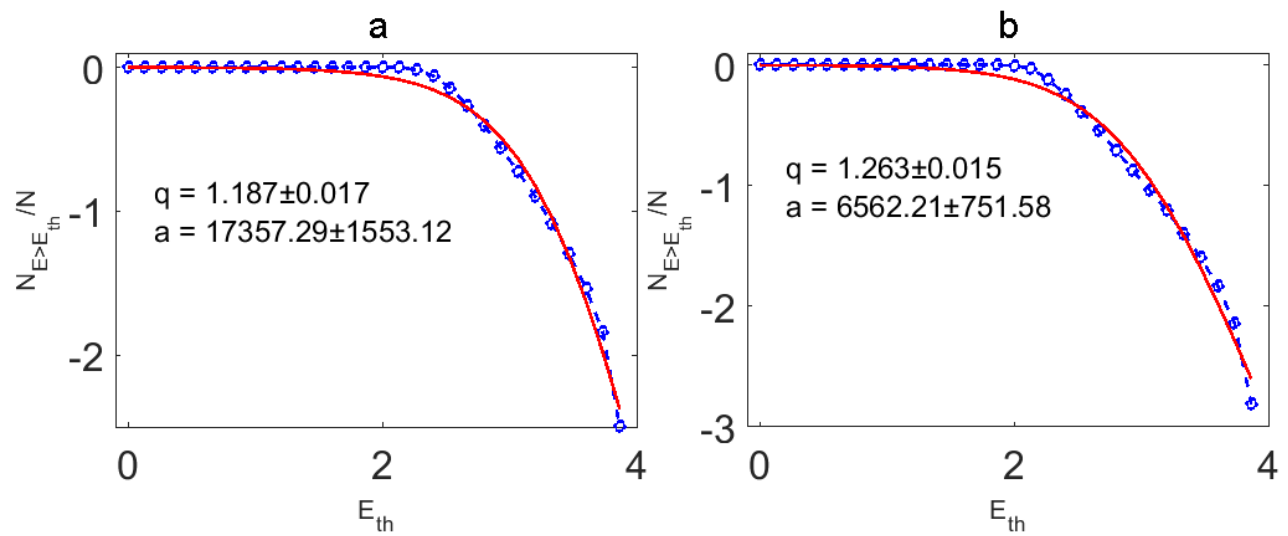

Fig. 4. Examples of energy distribution functions of AE signals with linear a)) and stepwise bt) load change and approximation by function (7).

The obtained value for the energy distribution functions of AE events $q=1.187$ and $q=$ 1.263 characterize the behavior of the sample under uniaxial loading as a system with memory and long-range correlations. The calculated values of the Tsallis parameters $q$ and $\mathrm{a}$, the errors in determining these values, and the number of AE signals for different experimental sessions are given in the table.

Table.. Tsallis parameters $q$ and $a$ for different samples.

\begin{tabular}{|l|l|l|l|l|l|}
\hline Rock & $N$ & $q$ & err $_{q}$ & $a$ & err $_{a}$ \\
\hline Granite \#1 & 16365 & 1.208 & 0.018 & 4519.27 & 589.09 \\
\hline Granite \#2 & 162782 & 1.157 & 0.017 & 20917.75 & 1784.60 \\
\hline Granite \#3 & 66789 & 1.144 & 0.018 & 20334.35 & 1867.91 \\
\hline Marble \#1 & 700 & 1.25 & 0.022 & 1545.20 & 257.55 \\
\hline Marble \#2 & 76891 & 1.233 & 0.012 & 4229.12 & 433.01 \\
\hline Sandstone \#1 & 18298 & 1.188 & 0.015 & 18327.72 & 1475.48 \\
\hline
\end{tabular}




\begin{tabular}{|l|l|l|l|l|l|}
\hline Sandstone \#2 & 24965 & 1.187 & 0.017 & 17357.29 & 1553.12 \\
\hline Sandstone \#3 & 24707 & 1.188 & 0.016 & 18501.11 & 1547.93 \\
\hline Sandstone \#4 & 9193 & 1.164 & 0.017 & 22533.06 & 1848.061 \\
\hline Sandstone \#5 & 61711 & 1.180 & 0.017 & 20202.86 & 1715.53 \\
\hline Sandstone \#6 & 102098 & 1.255 & 0.014 & 7409.52 & 727.11 \\
\hline Sandstone \#7 & 120697 & 1.248 & 0.016 & 8903.89 & 974.92 \\
\hline Sandstone \#8 & 45087 & 1.263 & 0.015 & 6562.21 & 751.58 \\
\hline
\end{tabular}

The calculated values of $q$ do not depend on the scenario of changing the load (Fig. 4), nor on the size or material of the sample, or on the number of events (see table) and have a value of $q \sim 1.2$.

The behavior of the Tsallis parameters $q$ and $a$, as well as the accumulation of AE signals and the AE activity throughout the experiment, are shown in Fig. 5, sample sandstone, loading - uniaxial, linear increasing. To plot the change in the Tsallis parameters $q$ and $a$, a sliding window of $500 \mathrm{AE}$ events with a shift of 1 event was used.

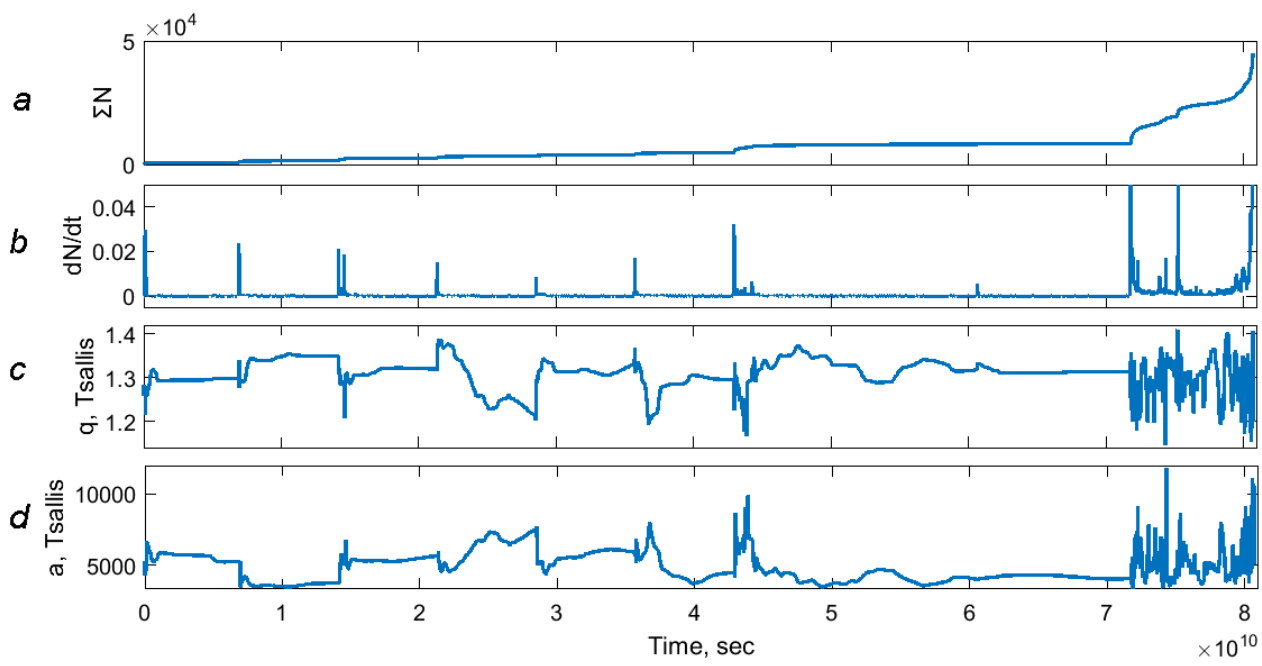

Fig. 5. Sample - sandstone, loading - linear. a - accumulation of AE events, b - activity of AE events, c - Tsallis parameter $q, \mathrm{~d}$ - Tsallis parameter $a$.

The accumulation of AE events occurs monotonically throughout the entire experiment, except for the stage immediately before destruction. The value of the Tsallis parameter $q$, as already described above, displays the measure of instability of the seismically active zone. a change in the parameters $q$ and a is observed with a sharp increases in the AE activity. Moreover, an increase in the AE activity is almost always accompanied by an increase in the value of the parameter q (Fig. 4c). This may mean an increase in long-range spatial correlations between individual recorded acts of microfracture of the sample.At the same time, the behavior of the parameter $a$ is of interest, which has the dimension of the volumetric energy density and is determined by the earthquake energy $E$ and the size of the fragment of blocks $r^{3}$ between the faults [38]. With an increase in the AE activity, this parameter (Fig. 4d) tends to increase. By analogy with earthquake aftershocks, an increase in the parameter $a$ can mean an avalanche-like formation of defects in a certain local region of the sample. When approaching the point of destruction, an increase in the number of AE events, an increase in AE activity, is also accompanied by significant changes in the Tsallis parameters $q$ and $a$. 


\section{Conclusion}

The work considers AE signals recorded under uniaxial compression of specimens of various geomaterials: sandstone, marble, granite. The logarithm of the maximum amplitude of the waveform of the recorded AE signal is conventionally taken as the energy of the AE signal.To describe the energy distribution function of AE signals, we used the provisions of nonequilibrium thermodynamics with the use of Tsallis statistics, which generalizes classical thermodynamics to the case of nonextensive systems. It is shown that the AE pulses flow is a system with memory and long-range spatial correlations. The change in the activity of the AE and the Tsallis parameters $q$ and $a$ in the sliding window throughout the experiment are considered. An increase in the AE activity is almost always accompanied by an increase in the value of the parameter $q$.

\section{Financing sources}

The research was partially carried out within the framework of the state assignment of the Federal State Budgetary Institution of Science of the Scientific Station of the Russian Academy of Sciences in Bishkek (theme№ AAAA-A19-119020190064-9).

\section{References}

1. L.M. Bogomolov, V.N. Sychev, B.Ts. Manzhikov, P.V. Ilyichev Geology and Geophysics vol 210 pp 1690-1696 (2001)

2. B.Ts. Manzhikov, V.A. Mansurov, V.S. Kuksenko, V.N. Saveliev Physics and mechanics of rock destruction Stavrogin A N (Frunze: Ilim) pp 77-84 (1983)

3. V.B. Smirnov, A.V. Ponomarev Izvestiya. Physics of the solid Earth 10 pp 26-36 (2004)

4. V.B. Smirnov, A.V. Ponomarev, P. Bernard, A.V. Patonin Izvestiya. Physics of the solid Earth 2 pp 17-49 (2010)

5. V.I. Vettegren, V.S. Kuksenko, M.A, Kryuchkov Izvestiya. Physics of the solid Earth 9 pp 40-45 (2006)

6. A.S. Zakupin, A.V. Aladiev, L.M. Bogomolov, B. V. Borovsky, P.V. Ilyichev, V.N. Sychev, N.A. Sycheva Volcanology and seismology 6 pp 22-33 (2006)

7. M.V. Gzovsky Fundamentals of Tectonophysics (Moscow: Nauka) p 535 (1975)

8. S.D. Vinogradov Acoustic method in research in the physics of earthquakes (Moscow: Nauka) p 177 (1989)

9. O.G. Shamina, V.I. Ponyatovskaya Modeling studies of heterogeneous and fractured media (Moscow: Institute of Physics of the Earth RAS) p 179 (1993)

10. G.A. Sobolev, A.V. Ponomarev, A.V. Koltsov Izvestiya. Physics of the solid Earth 12 pp 72-78 (1995)

11. V.A. Sinners, Yu.B. Drobot Acoustic emission (Moscow: Publishing house of standards) p 272 (1976)

12. V.I. Ivanov Flaw detection 5 pp 65-84 (1980)

13. M.A. Sadovskii, L.G. Bolkhovitinov, V.F. Pisarenko Deformation of the geophysical environment and seismic process (Moscow: Nauka) p 101 (1987)

14. M.B. Geilikman, V.F. Pisarenko On self-similarity in geophysical phenomena (Moscow: Nauka) pp 109-131 (1989)

15. G.G. Kocharyan, A.A. Spivak Deformation dynamics of block rock massifs (Moscow: Akademkniga) p 423 (2003)

16. G.A. Sobolev, A.V. Ponomarev Russian Journal of Earth Sciences vol 15 (1999)

17. O.G. Shamina Bulletin of the Academy of Sciences of the USSR. Geophysics Series 5 pp 513-518 (1956) 
18. K. Mogi Bull.Earthq.Res.Inst. vol 401 pp 125-173 (1962)

19. K. Mogi Bull. Earthq. Res. Inst. vol 404 pp 815-868 (1962)

20. S.D. Vinogradov, K.M. Mirzoev, N.G. Salomov Investigation of the seismic regime during sample destruction (Dushanbe: Donish) p 114 (1975)

21. S.D. Vinogradov Acoustic method in research in the physics of earthquakes (Moscow: Nauka) p 177 (1989)

22. E.E. Damaskinskaya, V.S. Kuksenko, N.G. Tomilin Construction of models of the development of the seismic process and earthquake precursors (Moscow: IFZ) vol 11 pp 9-16 (1993)

23. D.L. Lockner, J.D. Byerlee, V.S. Kuksenko et al. Fault Mechanics and Transport Properties of Rocks (London: Academic Press) pp 3-31 (1992)

24. B. Gutenberg, C.F. Richter Bull. of the Seismological Society of America vol $34 \mathrm{pp}$ 185-188 (1944)

25. N.V. Sarlis, E.S. Skordas, P.A. Varotsos Physical Review E August American Physical Society (APS) (2010)

26. K. Kasahara Mechanics of earthquakes (Moscow: World) p 264 (1985)

27. J.V. Gibbs Thermodynamics. Statistical mechanics (Moscow: Nauka) p 584 (1982)

28. C. Tsallis J. of Statistical Physics vol 52(1-2) pp 479-487 (1988)

29. O.V. Chumak Entropy and Fractals in Data (Izhevsk: Research Center "Regular and Chaotic Dynamics) p 164 (2011)

30. L. Telesca, C.C. Chen Nat. Hazards Earth Syst. Sci. vol 10 pp 1293-1297 (2010)

31. L. Telesca Tectonophysics vol 494 pp 155-162 (2010)

32. Chochlaki Kalliopi Entropy 20 p 721 (2018)

33. O. Sotolongo-Costa, A. Posadas Physical Review Letters February vol 924 (2004)

34. S.R. Brown, C.H. Scholz, J.B. Rundle Geophys. Res. Lett. vol 182 pp 215-218 (1991)

35. R. Silva, G.S. Franca, C.S. Vilar, J.S. Alcanis Phys. Rev. E. vol 73026102 (2006)

36. L. Telesca Entropy vol 13(7) pp 127-1280 (2011)

37. F. Vallianatos, G. Michas, G. Papadakis Physica A: Statistical Mechanics and its Applications vol 41415 November pp 163-173 (2014)

38. Complexity of Seismic Time Series: Measurement and Application Edited by Tamaz Chelidze Filippos Vallianatos Luciano Telesca 2016 Amsterdam Netherlands: Elsevier p 548 (2018)

39. G. Papadakis, F. Vallianatos, P. Sammonds Tectonophysics vol 608 pp 1037-1048 (2013)

40. S.M. Valverde-Esparza, A. Ramirez-Rojas, E.L. Flores-Marquez, L. Telesca. Acta Geophys 60 pp 833-845 (2012)

41. E.E. Damaskinskaya, I.A. Panteleev, D.I. Frolov, N.F. Vasilenko Geosystems of Transition Zones vol 23 pp 245-251 (2018)

42. I.P. Shcherbakov, V.S. Kuksenko, A.E. Chmel JETP Letters vol 945 pp 410-413 (2011)

43. O.B. Naimark Physical Mesomechanics vol 183 pp 71-83 (2015)

44. S. Zakupin, B.V. Borovskiy Patent 2012148683/28 RF BI 5 (2015)

45. D.S. Kulkov, S.A. Imashev Bulletin of the Kyrgyz State Technical University vol 2-1 50 pp 274-280 (2019) 